\title{
Algoritmos de aprendizaje automático de vanguardia para el diagnóstico de enfermedades
}

\author{
José Carlos de Jesús Montero Rodríguez, Rajesh Roshan Biswal, \\ Eddy Sánchez de la Cruz \\ Instituto Tecnológico Superior de Misantla \\ Misantla, Veracruz, México \\ 182T0885@itsm.edu.mx, rroshanb@itsm.edu.mx, esanchezd@itsm.edu.mx
}

\begin{abstract}
Resumen. En el presente trabajo se analizaron artículos sobre el uso de sistemas de aprendizaje automático utilizados para el diagnóstico de enfermedades como: cáncer de mama, cáncer de próstata, cardiovasculares, hipertensión, Parkinson, infartos, Artritis reumatoide, Triage, etc. También para la predicción de mortalidad en los hospitales y supervivencia posterior a eventos cardiovasculares, De los cuales se seleccionaron los más relevantes ya que en ellos los autores comparan el uso de diferentes algoritmos y herramientas de desarrollo dando su punto de vista de los más óptimos y con mayor índice de exito para realizar diagnósticos basados en base de datos, antecedentes e imágenes de acuerdo sus necesidades y tipos de enfermedades.
\end{abstract}

Palabras clave: aprendizaje automático, algoritmos, médico, diagnóstico.

\section{Advanced Machine Learning Algorithms for Disease Diagnosis}

\begin{abstract}
In the present work, articles regarding the use of machine learning systems used for the diagnosis of diseases such as breast cancer, prostate cancer, cardiovascular diseases, hypertension, Parkinson's, heart attacks, rheumatoid arthritis, triage, etc. were analyzed. Also for the prediction of the mortality and survival rate after cardiovascular events, the most relevant cases were selected where the authors compare the use of different algorithms and development tools. giving their point of view of the most optimal and with a higher rate of satisfaction to make diagnoses based on data base, background and images according to their needs and types of diseases.
\end{abstract}

Keywords: machine learning, algorithms, medic, diagnostic.

\section{Introducción}

El diagnóstico médico es una de las tareas más complejas de realizar ya que se requiere del análisis de muchos factores como: Anamnesis (es la información 
proporcionada por el paciente durante la entrevista clínica útil para analizar su situación clínica), síntomas, signos, exploración física[1]. El análisis de dichos factores en conjunto con la experiencia del médico da como resultado un diagnóstico el cual puede ser presuntivo o definitivo y en ciertos casos tiene que ser confirmados por exámenes médicos para llegar a un diagnóstico definitivo y poder tratar dicha afección. Por lo tanto, el uso de la tecnología para facilitar el diagnóstico de enfermedades es una necesidad en el siglo XXI ya que la creciente población y demanda de los servicios médicos sobrepasa la capacidad de atención de los hospitales dando como resultado tiempos de espera más largos y menos tiempo para la atención del paciente. El uso los Algoritmos de aprendizaje automático para el diagnóstico de enfermedades pueden reducir los tiempos de consulta e incluso en afecciones muy comunes evitar la consulta con el especialista ya que por medio de los sistemas se podría dar un diagnóstico certero y su tratamiento óptimo, incluso en las zonas geográficas más marginadas que no cuenta con especialistas un sistema manejado por un técnico de la salud podría diagnosticar y tratar dichas enfermedades sin la necesidad de que el paciente deje su zona.

En la última década, la tendencia de usar Algoritmos de aprendizaje automático se ha popularizado brindando muchas posibilidades para la toma de decisiones autónomas. En las áreas de la salud teniendo una notable evolución a pesar de que son áreas muy especiales debido a lo complejo que es tratar con la integridad de un ser humano, recalcando que cualquier error puede ser fatal para la salud del individuo pudiendo hasta costarle la vida.[2]

Dejando eso a un lado la evolución de los algoritmos en los últimos años han contribuido a mejorar el diagnóstico de enfermedades muy difíciles de diagnosticar como los desórdenes mentales, alzheimer e incluso poder predecir enfermedades cardiovasculares que de la manera tradicional no se podrían predecir con la misma efectividad[1]. La finalidad del siguiente trabajo es contrastar los diferentes algoritmos y herramientas utilizados por los autores en sus casos de estudios particulares donde podemos observar el índice de efectividad de los diferentes algoritmos tales como redes neuronales, redes bayesianas, K-means, razonamiento basado en casos y el uso de herramientas como weka.

\section{Metodología}

Se realizó una búsqueda en bibliotecas y revistas virtuales: Rev Ciencias Médicas, Google scholar, revista espacios, Pistas Educativas, European Journal of Internal Medicine. De los cuales se seleccionaron artículos literarios que no fueran mayores a 10 años de antigüedad y donde los autores abordaran el uso de algoritmos de aprendizaje automático para el diagnóstico clínico y la predicción de enfermedades a través de información brindada por los pacientes, estudios clínicos, historias clínicas y casos médicos en los cuales se pudiera cuantificar los resultados para evaluar el desempeño de los diferentes algoritmos utilizados en cada estudio a su vez se descartaron los artículos que no presentaban resultados sólidos y solo hacían propuesta de la importancia del uso de algoritmos de 
aprendizaje automático para el diagnóstico médico. Para el control de la calidad de los artículos y de su información se tomó en cuenta si estaban publicados en alguna revista científica o sitio en línea con buena reputación. Se seleccionaron artículos muy variados en cuanto a las enfermedades que diagnosticaban ya que con ello se puede cuantificar cual de los algoritmos tienen mejor desempeño en diversas áreas de la salud.

\section{Desarrollo}

\subsection{Tipos de algoritmos de aprendizaje automático para el diagnóstico de enfermedades}

EL diagnóstico de enfermedades es un proceso cognitivo complejo que implica capacitación, experiencia, reconocimiento de patrones y cálculo de probabilidad condicional, entre otros componentes menos comprendidos. En las últimas décadas se han realizado varios esfuerzos por aplicar el análisis predictivo en los sistemas de salud, así como lanzar sistemas de aprendizaje automático para facilitar el diagnóstico[1]. Los algoritmos de aprendizaje automático son un área del conocimiento donde convergen diferentes técnicas, en particular la redes neuronales artificiales (RNA), los árboles de decisión (AD) Y las redes bayesianas, teniendo un gran desarrollo e impacto en diversas áreas del conocimiento, incluida la medicina. Los métodos estudiados se han utilizado en medicina con varios propósitos: clasificación de diagnóstico de enfermedades y predicción de la mortalidad de diversas patologías[1]:

1. Las redes neuronales artificiales,

2. El razonamiento basado en casos,

3. Las redes bayesianas,

4. Árboles de decisión.

Las redes neuronales artificiales han demostrado que producen buenos resultados en bases de datos con muchas instancias. Las redes neuronales no consideran la incertidumbre, actúan como una caja negra, en el sentido de que no es posible saber cómo se ha llegado a los resultados obtenidos y los nodos intermedios no pueden ser interpretados[1].

El razonamiento basado en casos se ha utilizado con éxito en el diagnóstico médico asistido, esta técnica se basa en el paradigma de resolución de problemas en el que un problema nuevo se soluciona atendiendo casos similares del pasado. El ejemplo más conocido, es el vecino más cercano, que consiste en comparar cada caso problema con una serie de casos almacenados; el caso problema nuevo y los casos almacenados consisten en un número de atributos o variables y éstos se comparan para encontrar el caso almacenado que más se parece al caso problema, de esta manera, se puede seleccionar la enfermedad conocida que más se parezca a la descripción del paciente. Una vez resuelto, este nuevo caso pasa a formar parte de la biblioteca de casos disponibles para comparar, con lo que el sistema se vuelve cada vez más sólido[1]. 
Las redes bayesianas calculan la probabilidad de que un caso con cierto atributo pertenezca a una categoría. En medicina se han usado para predecir recaída de enfermedad o riesgo de cáncer. El filtro no sabe esto por adelantado y tiene que aprender de la mano del usuario. El programa calcula en lo sucesivo la probabilidad de que cada atributo corresponda a una categoría o a otra. Basado en las características que se describen sobre las técnicas de IA que con frecuencia se utilizan para el diagnóstico de enfermedades, se persigue como objetivo comparar dichas técnicas para seleccionar la que mejor se ajusta al diagnóstico de enfermedades, cuando se tienen datos almacenados sobre el comportamiento de las enfermedades que con frecuencia afectan a una población en cuestión[1].

El árbol de decisión es un diagrama que representa en forma secuencial condiciones y acciones, normalmente construidos a partir de la descripción de la narrativa de un problema. Ellos proveen una visión gráfica de la toma de decisión necesaria, especifican las variables que son evaluadas, qué acciones deben ser tomadas y el orden en el cual la toma de decisiones será efectuada, y permite que quien las toma descomponga un problema complejo y grande, en varios más pequeños, proporcionando un método efectivo para la toma de decisiones debido a que: plantea el problema para que todas las opciones sean analizadas, permite considerar totalmente las posibles consecuencias de tomar una decisión y ayuda a realizar las mejores medidas sobre la base de la información existente y de las mejores suposiciones. Al tener en cuenta todos estos elementos, se decidió utilizar la técnica de árbol de decisiones como vía para garantizar un diagnóstico eficiente y ágil, lo que permitirá trabajar con metas más elevadas y necesarias como el diagnóstico en etapas tempranas de la enfermedades [27].

\subsection{Algoritmos aproximados empleados en problemas de clasificación}

Perceptrón multicapa (Multi-Layer Perceptron, MLP). Un MLP es una red feed-forward con aprendizaje supervisado. El tipo de aprendizaje más utilizado por un perceptrón es el método backpropagation (propagación del error hacia atrás) basado en la generalización de la regla Delta. A pesar de sus limitaciones, este método ha contribuido a ampliar de forma considerable el rango de aplicaciones de los MLPs (diágnóstico de enfermedades, clasificación de señales, simulación de la dinámica del brazo de un robot, puntuación para la solicitud de créditos, etc.). En este tipo de aprendizaje, primero se aplica un patrón de entrada como estímulo a la primera capa de las neuronas de la red, el cual se propaga a través de todas las capas ocultas hasta generar una salida (paso forward). Dicha propagación se lleva a cabo al aplicar una función de activación a la suma ponderada, que surge de la combinación lineal de las entradas y las conexiones (o pesos) sinápticos correspondientes. Luego se evalúa el error cometido al comparar el resultado obtenido por las neuronas de salida con el patrón de salida, por medio de una función de costo.

A continuación, estos errores se transmiten hacia atrás (paso backward), partiendo de la capa de salida hacia todas las neuronas de la capa intermedia que contribuyan directamente a la salida. Este proceso se repite, capa por capa, 
hasta que todas las neuronas de la red hayan recibido un error que describa su aporte relativo al error total. En base a esto, se reajustan los pesos de conexión de cada neurona, de manera que la próxima vez que se presente el mismo patrón, la salida esté más cercana a la deseada. Para acelerar el proceso de aprendizaje se puede añadir un término denominado momento o momentum, el cual acelera la convergencia del algoritmo. Otra forma de mejorar el proceso de aprendizaje es adaptar la tasa de aprendizaje, $n$, durante dicho proceso, conocido como $\mathrm{n}$ adaptativo, y consiste en modificar el valor de n durante el entrenamiento con el fin de evitar que el error se estanque debido a la presencia de óptimos locales [24].

Algoritmos Genéticos (Genetic Algorithms, GAs). Los GAs son un tipo popular de Metaheurísticas, en especial de algoritmos evolutivos, que simula la evolución de individuos por medio del proceso de selección natural Darwiniano. Computacionalmente hablando, esto se refiere a que realizan una búsqueda multidireccional manteniendo una población de potenciales soluciones (individuo) y fomentando, por un lado, la formación de información (por medio de la mutación), y por otro lado, el intercambio de información (por medio del crossover) entre dichas soluciones. La selección de los individuos a reproducirse (crossover y mutación), se realiza a través de operadores de selección probabilísticos (ruleta, ranking, torneo binario, entre otros). Generación tras generación, los individuos de la población actual son sustituidos por los recién creados. Este reemplazo usualmente intenta mantener los mejores individuos (elitismo) eliminando los peores. Dependiendo de si se tiene en cuenta la antigua población se pueden obtener dos tipos de estrategias de reemplazo: $(\mu ; \lambda)$ si el reemplazo se realiza utilizando únicamente a la población de hijos, $\mathrm{y}(\mu+\lambda)$ si el reemplazo $\mu$ se realiza seleccionando individuos de la unión de la población antigua y la de hijos. Los dos GAs propuestos, GA1X y GA2X, utilizan números reales en la representación de cada individuo, la selección de los padres se realiza por medio de un torneo binario, método de selección que es utilizado también en el proceso de reemplazo $(\mu+\lambda)$ con elitismo[25]. Además aplican el método swap de un gen (elemento de la solución) elegido al azar para llevar a cabo la mutación de un individuo bajo una determinada probabilidad (Pm)[25]. La diferencia entre GA1X y GA2X radica en el método de crossover empleado. Por un lado, GA1X utiliza crossover de un punto, con el fin de testear un GA estándar. En tanto que, GA2X aplica el crossover de dos puntos, cuyo objetivo es introducir mayor variación en cada descendencia generada. Ambos operadores se aplican bajo una cierta probabilidad (Pc).

El origen de los genes de cada individuo; donde cada uno se compone de los pesos sinápticos de un MLP. Los individuos se estructuran en forma de vector, a diferencia de la estructura matricial utilizada en los MLPs. En otras palabras, cada peso de conexión representa un gen del individuo, siendo creados con valores aleatorios en el rango [-0.5, 0.5] siguiendo una distribución uniforme[25].

El fitness mide la capacidad del individuo para resolver los problemas planteados, es decir, el promedio de error cometido al intentar clasificar patrones de cáncer de mama o diabetes. Mientras menor sea dicho error, el fitness del indi- 
viduo será mejor. En otras palabras, la evaluación del fitness de cada individuo se corresponde con el cálculo del error medio obtenido al ejecutar sólo el paso hacia adelante del MLP durante una época [25].

\section{Estado del arte}

Para el diagnóstico de patologías presentes en la columna vertebral se utilizó el software weka en conjunto con: algoritmos de clasificación como LMT, Redes Bayesianas, Naive Bayes, J48 y Naive Bayes Simple para decidir cuál es el mejor de ellos para el diagnóstico de dicha enfermedad. Los resultados obtenidos durante la clasificación de los datos por medio de los algoritmos utilizados muestran que el algoritmo de decisión LMT clasificó 265 instancias correctamente y solo 45 instancias incorrectamente de un total de 310 instancias con un porcentaje de éxito de $85.483 \%$, y un error absoluto de 0.2168. El algoritmo Redes Bayesianas clasificó correctamente 237 de las 310 instancias con un porcentaje de éxito de $80 \%$ y un error absoluto de 0.249; el algoritmo Naive Bayes clasificó correctamente 248 instancias con un error absoluto de 0.2, mientras que J48 clasificó 253 instancias, finalmente el algoritmo Naive Bayes Simple clasificó correctamente 241 instancias de las 310, llegando a la conclusión de que el mejor algoritmo de decisión es el LMT[3]. Un perceptrón fue utilizado con el objetivo principal de diseñar un método no invasivo para el diagnóstico de pacientes con cirrosis hepática utilizando únicamente datos de laboratorios. Como resultado del estudio tras comparar las RNA en término del rendimiento, el modelo fue capaz de reconocer 3 tipos de valores: cirrosis, no cirrosis y los no identificables con un tasa de éxito de casi el 90\%[4].

En otras áreas del campo médico se han propuesto las RNA para pronosticar la estancia hospitalaria prolongada de pacientes mayores en el servicio de urgencias, donde el mejor desempeño fue logrado por el MLP con una sensibilidad del $62.5 \%$ y especificidad del $96.6 \%$ [5]; asimismo, pueden predecir la estancia prolongada en la unidad de cuidados intensivos y la mortalidad con la ayuda de algoritmos como, el vecino más cercano (K-NN), SVM, árboles de clasificación (CART), entre otros. Los datos mostraron que el modelo SVM ofreció los mejores resultados con un valor del área bajo la curva de 0.77 para predecir la estancia y del 0.82 para la predicción de la mortalidad [6]. Por otra parte, en la investigación del cáncer han sido aplicadas por su habilidad para reconocer patrones complejos como, en la predicción de cáncer avanzado de vejiga en pacientes sometidos a cistectomía radical[7], cáncer de mama, el modelo fue evaluado utilizando la matriz de confusión y las curvas ROC. Con los datos de prueba, el modelo RNA implementado dió como resultado una tasa de predicción de la enfermedad del $90.5 \%$. Con estos valores predictivos se concluye que el modelo RNA es rápido, fiable y de gran ayuda en esta especialidad médica[8], además puede predecir la supervivencia después de la recesión hepática por cáncer colorrectal. La investigación comparó el uso de las RNA con el modelo de regresión C-index encontrando que a través del análisis de 241 pacientes y de la selección de seis variables de entrada de 28 . El modelo RNA predice la supervivencia individual 
con mayor exactitud que el modelo de regresión de Cox, con de 0.72 frente a 0.66 [9]. Igualmente han sido utilizadas con gran éxito en predicción de mortalidad. Por ejemplo, la mortalidad hospitalaria de los pacientes con reparaciones de aneurisma aórtico abdominal roto, en el cual la RNA mostró mejor desempeño cuando esta fue comparada con los resultados obtenidos por la metodología de regresión lineal[10]. En el pronóstico de la mortalidad de cáncer de vejiga en este trabajo se utilizaron siete métodos de aprendizaje para predecir la mortalidad a los cinco años después cistectomía radical, incluyendo a la Red neuronal MLP, redes de función de base radial, la máquina de aprendizaje extremo EML, regularizados ELM (RELM), SVM, las RB naive y el clasificador del vecino más cercano $(\mathrm{K}-\mathrm{NN})$. Los resultados en Experimentales indican que RELM logra la más alta precisión en la predicción con un 0.8 . Los resultados de la investigación demuestran el potencial de la aplicación de esta técnica de aprendizaje para apoyar la toma de decisiones clínicas[11]; en el cáncer de páncreas el estudio utilizó las RNA para predecir la supervivencia individual a largo plazo de los pacientes sometidos a cirugía radical en el tratamiento de este tipo de cáncer. Los resultados fueron comparados con el modelo de regresión C-index mostrando que las RNA tiene mejor desempeño con un 0.79 , mientras que el otro modelo mostró un 0.67 de precisión[12]. En pacientes con accidente cerebrovasculares (isquemia y hemorragia) para el pronóstico de la mortalidad a los 10 días después del evento. La investigación aplicó redes MLP y los métodos estadísticos multivariable (Análisis discriminante multivariado [MDA] y análisis de regresión logística [LRA]. El MLP tiene una la clasificación del 100\%, para ambos grupos isquémicos y hemorrágicos en la fase de entrenamiento. Los métodos LRA y MDA mostraron resultados exitosos, a pesar de que estos resultados son más bajos el MLP tanto para el grupos hemorrágico y como para el grupo isquémico en la fase de entrenamiento. Sin embargo, cuando se compararon los resultados de los métodos en la fase de prueba, la mejor clasificación, especificidad, sensibilidad y precisión del $87.8 \%$ fue alcanzado por el modelo MDA para el grupo hemorrágico. La mejor clasificación, especificidad $75.9 \%$, sensibilidad $85.9 \%$ y precisión $80.9 \%$ para el grupo isquémico fue alcanzado por la técnica LRA[13].

Por otro lado el estudio de colak y cols (2015)[14] mostró buenos resultados en la predicción de accidentes cerebrovasculares aplicando el MLP, las máquinas de vectores y las RBF, los cuáles mostraron una precisión del $80 \%$. Las RPF también ha mostrado un buen desempeño en el diagnóstico de enfermedades del corazón[15], en el diagnóstico y análisis de la fibrilación articular[16]. Debido a su excelente desempeño el tratamiento de imágenes las redes SOM han aplicado en la segmentación de imágenes médicas (segmentación de tumores de mamas[17] y cerebrales[18]), además para la identificación de desórdenes como la demencia, esquizofrenia o el alzheimer[19]. Los AD que tienen entre otras características su aporte visual para la toma de decisiones, han sido utilizados para identificar los factores de riesgos asociados con la hipertensión arterial. Los modelos fueron evaluados con 9078 sujetos divididos en dos grupos (hipertensos y no hipertenso). Los resultados para el primer modelo fueron $73 \%$ de sensibilidad, $63 \%$ especificidad y $77 \%$ de precisión. Los valores correspondientes para el segundo 
modelo fueron $70 \%, 61 \%, 74 \%$ respectivamente. Según los autores el estudio proporcionó reglas de clasificación fáciles y comprensibles para identificar los factores de riesgo asociado con la hipertensión y esto puede ser útil para el desarrollo de programas para el manejo de hipertensión[20]. Cómo ayuda en la toma de decisiones en paciente con soporte ventricular izquierdo, se estudiaron 183 pacientes con fallas ventriculares derechas, de los cuales 27 requirieron soporte ventricular izquierdo, los restantes continuaron con manejo médico hasta el trasplante o fallecimiento. El modelo identificó 8 variables preoperatorias óptimas. El árbol de decisiones resultante que constaba de 28 ramas y 15 hojas, identificó a los pacientes que necesitaban soporte ventricular con una sensibilidad del $85 \%$ y un área bajo la curva de 0.87[21]. En el pronóstico del cáncer pleural[22], además, en la predicción de los síntomas de Parkinson[23].

\section{Estudios y contribuciones}

Araújo, A. et al. Comparar diferentes algoritmos de aprendizaje automático para la clasificación de quistes mamarios a través de imágenes termográficas usando redes neuronales artificiales, árboles de decisiones, clasificadores bayesianos y atributos de Haralick y Zernike. La base de datos de imágenes está compuesta por termografía, las imágenes fueron adquiridas en el Hospital Universitario de la Universidad Federal de Pernambuco. Estas imágenes son clínicamente clasificadas en las clases quistes malignos y benignas. Momentos de Zernike y Haralick fueron utilizados como atributos. Las máquinas de aprendizaje extremo (ELM) y las redes de Perceptrón multicapa (MLP) demostraron ser bastante eficientes clasificadores para la clasificación de lesiones mamarias en imágenes termográficas. Utilizando el $75 \%$ de la base de datos para la formación, el valor máximo obtenido por precisión fue de $73.38 \%$, con un índice Kappa de 0.6007 . Este resultado indica a un Sensibilidad del $78 \%$ y especificidad del $88 \%$. La eficiencia global del sistema fue del $83 \%$. Conclusión: ELM mostró ser un clasificador prometedor para ser usado en la diferenciación de lesiones de mama en imágenes termográficas, debido a su bajo coste computacional y robustez[26].

González, F., Análisis de diferentes artículos sobre Machine learning models in rheumatology y cómo evolucionaron desde los años 1989 hasta el 2015 sintetizando los algoritmos más con mejores resultados para el diagnósticos de enfermedades en esta rama de la medicina con el uso aprendizaje supervisado como las redes neuronales y las redes bayesianas, y de aprendizaje no supervisado como el método de agrupamiento k-medias. Adicionalmente, para la identificación de endofenotipos los autores utilizaron alineamiento de secuencias de aminoácidos. Los resultados presentados en el artículo son bastante prometedores: los modelos predictivos logran un alto desempeño en la tarea de discriminar entre pacientes con artritis reumatoide y controles, con sensitividad y especificidad de hasta el $92,3 \%$ y el $93,3 \%$, respectivamente. Por otro lado, el análisis de conglomerados basado en k-medias permitió establecer 2 grupos de pacientes con diferentes niveles de severidad. Finalmente, el análisis de secuencias de aminoácidos reveló secuencias comunes en pacientes con desenlaces iguales[28]. 
Morales, L., Comparación de modelos de inteligencia computacional para la clasificación e identificación de endofenotipos (relación entre fenotipo y marcadores genéticos) en pacientes con artritis reumatoide y controles sanos, a partir de información genética, principalmente el HLA DRB1 (antígeno leucocitario humano) y la teoría del epítope compartido. Esto hace referencia a la asociación entre la artritis reumatoide y el HLA DRB1, principalmente los alelos que contienen un motivo común de aminoácidos de las secuencias QKRAA, QRRAA o RRRAA en las posiciones 70 a 74 de la cadena del DRB1, los cuales confieren una susceptibilidad particular de la enfermedad a los individuos con técnicas de inteligencia computacional como son las redes neuronales, redes bayesianas y métodos como k-means. Como datos de entrada se utilizaron variables como: factor reumatoide, anticuerpos contra péptido citrulinado, proteína $\mathrm{C}$ reactiva, número de articulaciones inflamadas y dolorosas, rigidez matinal, edad, género, antecedentes de comorbilidades y la información del alelo HLA DRB1. Se utilizaron técnicas de bioinformática para la búsqueda de secuencias de aminoácidos relacionadas con la severidad de la enfermedad.

Se obtuvieron resultados importantes para el diagnóstico de la enfermedad, así como también para su categorización y como potencial aplicación en la medicina personalizada de los individuos afectados por esta enfermedad. Se diseñaron modelos computacionales para clasificación, dentro de los cuales la red neuronal utilizando 5 variables obtuvo una sensibilidad del $92,3 \%$ con una especificidad del 86,66\% y la red bayesiana logró una sensibilidad del 92,3\% y una especificidad del 93,33\%. La red neuronal utilizando 11 variables obtuvo una sensibilidad del $84,61 \%$ y una especificidad del $93,33 \%$ mientras que la red bayesiana consiguió una sensibilidad del $92,3 \%$ con una especificidad del $93,33 \%$. Un método de agrupamiento tipo k-means fue utilizado para dividir en 2 grupos la totalidad de pacientes y controles. Además, se logró separar 2 grupos entre los pacientes para definir su severidad. Finalmente, se consiguió obtener un árbol de distancia entre secuencias de aminoácidos de los distintos alelos HLA DRB1, lo cual permite visualizar grupos con cercanía genética y lograr determinar que posiblemente existen más grupos fuera de los propuestos en las distintas teorías[29].

María, M., Comparación de diferentes algoritmos de aprendizaje automático para el diagnóstico del triaje en el servicio de urgencias a través de 1000 casos para realizar le evaluación de los modelos, que será obtenida a partir de casos prácticos en salas de urgencias de hospitales de Buenos Aires (se encuentran entre los potenciales interesados el Hospital Posadas, Hospital de Haedo, Hospital Italiano, Hospital Alemán, entre otros). En el estudio realizado hasta el momento se ha hecho una simulación con 124 casos. Se han escogido 40 variables que han sido seleccionadas a criterio de los expertos. En la figura 2 (b) se puede ver el clasificador bayesiano aprendido a partir de los datos con el algoritmo K2 (Cooper y Herskovits, 92). Se trata de un algoritmo para el aprendizaje automático de redes bayesianas.

A partir de la red obtenida, el clasificador de la variable "triaje" puede ser obtenido con la sencilla selección de las variables que forman el manto de 
Markov (Sebastiani et al., 2005b) de dicha variable. Se ha utilizado el software Bayes Discoverer (Sebastiani et al., 2005b). En un segundo paso se ha utilizado directamente un clasificador bayesiano: el clasificador bayesiano simple, sobre las mismas 40 variables. Para analizar los datos de la simulación se han utilizado los siguientes algoritmos de aprendizaje automático: C45 (Quinlan, 1996): Basado en la creación de un árbol de decisión, C45 es el más aplicado entre los de su clase (C45). El vecino más cercano: Un algoritmo basado en instancias (Aha, et al., 1991). Se usarán los parámetros k=1 y k=5 (VMC1, VMC5). K2: Algoritmo genérico para creación de redes bayesianas. Clasificador bayesiano simple (Naive Bayes classifier): Específico para clasificadores, con estimación de máxima verosimilitud (BS0) y estimador bayesiana con factor $. \alpha .=1$ (BS1). De cada uno de ellos se ha obtenido la precisión (en porcentaje de aciertos) utilizando validación cruzada de 5 hojas. La figura 1 muestra la precisión para cada uno de los métodos empleados, ordenador por la misma. Como puede observarse, todos los algoritmos de redes bayesianas utilizados obtienen mejor precisión [30].

\begin{tabular}{|l|l|}
\hline Algoritmo & Precisión \\
\hline BS1 & $87,90 \%$ \\
\hline K2 & $86,99 \%$ \\
\hline BS0 & $86,23 \%$ \\
\hline C45 & $79,67 \%$ \\
\hline VMC5 & $80,63 \%$ \\
\hline VNC1 & $80,50 \%$ \\
\hline
\end{tabular}

Fig. 1. Resultado de los algoritmos para el diagnóstico del triaje en el servicio de urgencias [30].

J. John, Entrenamiento y prueba de distintas redes neuronales para el diagnóstico de infarto agudo de miocardio, se realizó el entrenamiento y la prueba de varias redes neuronales, con diferentes arquitecturas para el diagnóstico del infarto, a partir de los datos de la escala de clasificación de la probabilidad de angina de Braunwald en un grupo de pacientes que ingresaron por dolor torácico al servicio de urgencias del Hospital San José de Bogotá. Se generaron 40 redes que fueron probadas en 5 experimentos de los cuales se obtuvo mayor precisión diagnóstica con el modelo de 5 entradas electrocardiográficas más troponina, aunque el mejor valor predictivo negativo se alcanzó en el modelo con 10 variables clínicas, electrocardiográficas y troponina. Varias de las redes diseñadas tuvieron una sensibilidad y una especificidad del $100 \%$. Se requiere un estudio de validación para comprobar estos hallazgos[31].

\section{Análisis de los algoritmos de aprendizaje automático}

Con la revisión de los trabajos anteriormente mencionados se puede tener una visión de cuáles son los algoritmos que tiene un mejor desempeño para 
el diagnóstico de enfermedades, teniendo en cuenta que dichos valores varían dependiendo de la enfermedad a diagnosticar como se muestra a continuación en la figura 2 seleccionando el los mejor algoritmos para cada enfermedad.

\begin{tabular}{|c|c|c|}
\hline Enfermedad & Algoritmos & Tasa de éxito \\
\hline Patologias de columna vertebral & LMT & $85.48 \%$ \\
\hline Cirrosis hepática & RNA & $90 \%$ \\
\hline Cáncer de mama & RNA & $90.5 \%$ \\
\hline Aneurisma aórtico abdominal roto & RNA & $84 \%$ \\
\hline Fibrilación auricular & RBF & $95 \%$ \\
\hline Artritis reumatoide & RB & $93.33 \%$ \\
\hline Hipertensión arterial & AD & $77 \%$ \\
\hline Diagnóstico del triaje & BS1 & $87.90 \%$ \\
\hline
\end{tabular}

Fig. 2. Resultado de los algoritmos para el diagnóstico de enfermedades.

Haciendo un comparación de los artículos evaluados observamos que la mayoría de los autores que usan RNA para el diagnóstico de enfermedades y predicciones de mortalidad obtiene un mejor desempeño con la RNA. El acercamiento a la metodología basada en redes neuronales artificiales puede hacerse con dos perspectivas. Por una parte, desde sus ventajas: las redes son capaces de trabajar sin las restricciones de los modelos estadísticos detectando las relaciones no lineales y las interacciones entre las variables predictoras. Y por otra, asumiendo sus desventajas: mayor complejidad de interpretación de sus parámetros de funcionamiento, mayor necesidad de recursos informáticos, alto componente empírico en su construcción y mayor dificultad de exportar el modelo para aplicarlo a otras poblaciones. Estas desventajas influyen en una menor difusión de la técnica para su uso habitual.

En general, una RNA es potencialmente más precisa que las técnicas estadísticas cuando la variable pronóstica se expresa como una función compleja de las variables predictoras o cuando existe interdependencia entre éstas; pero son estructuralmente complicadas y sus parámetros son más difíciles de interpretar.[32].

\section{Conclusión}

Como se observa el uso de algoritmos de aprendizaje automático para el diagnóstico de enfermedades a evolucionado de manera notable a lo largo de estos años destacando las RNA, RB, MLP y MLT siendo de gran utilidad para la predicción y el diagnóstico de enfermedades como cáncer de mama, cáncer de próstata, cardiovasculares, hipertensión, Parkinson, infartos. Aportando un 
apoyo para los médicos y los pacientes ya que gracias a estos se pueden prevenir y tratar adecuadamente antes de que presenten complicaciones mayores mejorando notoriamente las probabilidades de supervivencia en dichos casos. También han sido de ayuda en la área de urgencias de los hospitales al realizar el diagnóstico de los triajes de los pacientes que llegan y poder reducir tiempos de valoración para poder asignarles un número de atención correcto y optimizar el proceso de urgencias que es una de las áreas más de mandadas en los hospitales dejando los procesos rudimentarios que entorpecen la atención.

Por otra parte, evaluando los algoritmos y viendo sus resultados concluyendo de que uno de los modelos más óptimos para realizar dicha tarea es el MLP, sin dejar a un lado que al ser un área compleja y con muchas posibilidades pueda que en otros casos sean más óptimos otros algoritmos, dejando en claro que al momento de desarrollar un sistema de diagnóstico inteligente lo más viable es evaluar diferentes métodos de acuerdo a la afección que se desee predecir o diagnosticar.

Generando un interés para creación de sistemas que puedan ayudar a mejorar los tiempos de atención en los hospitales. Una propuesta seria la creación de un sistema experto para el diagnóstico de enfermedades obstétricas en el hospital regional de alta especialidad de la mujer para mejora la calidad de la atención y poder agilizar procesos ya que dicho hospital es el más importante de Tabasco y año con año su demanda aumenta y cada vez se hace más difícil la atención de los pacientes de manera eficiente generando situaciones particularmente complicadas.

\section{Referencias}

1. Gonzalez Benitez, N., Estrada Senti, V., Febles Estrada, A.: Estudio y selección de las técnicas de Inteligencia Artificial para el diagnóstico de enfermedades. Rev Ciencias Médicas, Pinar del Río 22(3), 131-141 (2018)

2. Leyva Vázquez, G.M., González Benítez, N., Hechavarría Hernández, J., Rivero Peña, Y., Daher Nader, J.E.: El diagnóstico de enfermedades desde el Análisis Inteligente de los Datos. Revista espacios 39(28), 16 (2018)

3. Ramírez Pérez, N.V., Laguna Estrada, M., De Anda Tovar, A.M.: Aplicación De Métodos De Inteligencia Artificial en el Área Médica. Pistas Educativas 35(111), $124(2015)$

4. Bostan, V.M., Pantelimon, B.: Creating a Model based on artificial neural nettwork for liver cirrhosis diagnostise. In: 9th International Symposioum on Advanced Topics in Electrical Engineering (ATEE), pp. 295-298 (2015)

5. Launay, C.P., Rivière, H., Kabeshova, A., Beauchet, O.: Predicting Prolonged Length of Hospital Stay in Older Emergency Department Users: Use of a Novel Analysis Method, the Artificial Neural Network. European Journal of Internal Medicine (2015)

6. Houthooft, R., Ruyssinck, J., Van der Herten, J., Stijven, S., Couckuyt, I., Gadeyne, B., Ongenae, F., Colpaert, K., Decruyenaere, J., Dhaene, T., De Turck, F.: Predictive Modelling of Survival and Length of Stay in Critically Ill Patients Using Sequential Organ Failure Scores. Artificial Intelligence in Medicine 63(3), 191-207 (2015) 
7. Vukicevic, A.M., Jovicic, G.R., Stojadinovic, M.M., Prelevic, R., Filipovic, N.D.: Evolutionary Assembled Neural Networks for Making Medical Decisions with Minimal Regret: Application for Predicting Advanced Bladder Cancer Outcome. Expert Systems with Applications 41(18), 8092-8100 (2014)

8. Saritas, I.: Prediction of Breast Cancer Using Artificial Neural Networks. Journal of Medical Systems 36(5), 2901-2907 (2012)

9. Spelt, L., Nilsson, J., Andersson, R., Andersson, B.: Artificial Neural Networks a Method for Prediction of Survival Following Liver Resection for Colorectal Cancer Metastases. European Journal of Surgical Oncology. The Journal of the European Society of Surgical Oncology and the British Association of Surgical Oncology 39(6), 648-54 (2013)

10. Wise, E.S., Hocking, K.M., Brophy, C.M.: Prediction of In-hospital Mortality After Ruptured Abdominal Aortic Aneurysm Repair Using an Artificial Neural Network. Journal of Vascular Surgery 62(1), 8-15 (2015)

11. Wang, G., Lam, K., Deng, Z., Choi, K.: Prediction of Mortality After Radical Cystectomy for Bladder Cancer by Machine Learning Techniques. Computer in Biology and Medicine 63(C), 124-132 (2015)

12. Ansari, D., Nilsson, J., Andersson, R., Regnér, S., Tingstedt, B., Andersson, B.: Artificial Neural Networks Predict Survival from Pancreatic Cancer After Radical Surgery. American Journal of Surgery 205(1), 1-7 (2013)

13. Çelik, G., Baykan, O. K., Kara, Y., Tireli, H.: Predicting 10-day Mortality in Patients with Strokes Using Neural Networks and Multivariate Statistical Methods. Journal of Stroke and Cerebrovascular Diseases. The Official Journal of National Stroke Association 23(6), 1506-1512 (2014)

14. Colak, C., Karaman, E., Turtay, M.G.: Application of Knowledge Discovery Process on the Prediction of Stroke. Computer Methods and Programs in Biomedicine 119(3), 181-185 (2015)

15. Alsalamah, M., Amin, S., Halloran, J.: Diagnosis of Heart Disease by Using a Radial Basis Function Network classification technique on patients' medical records. In RF and Wireless Technologies for Biomedical and Healthcare Applications (IMWSBio), IEEE MTT-S International Microwave Workshop Series on, pp. 1-4 (2014)

16. Mateo, J., Rieta, J.J.: Radial Basis Function Neural Networks Applied to Efficient QRST Cancellation in Atrial Fibrillation. Computers in Biology and Medicine 43(2), 154-163 (2013)

17. Torbati, N., Ayatollahi, A., Kermani, A.: An Efficient Neural Network Based Method for Medical Image Segmentation. Computers in Biology and Medicine 44, pp. 76-87 (2014)

18. Mei, P., de Carvalho Carneiro, C., Fraser, S.J., Min, L., Reis, F.: Analysis of neoplastic lesions in magnetic resonance imaging using selforganizing maps. Journal of the Neurological Sciences 359(1), 78-83 (2015)

19. Ortiz, A., Gorriz, J.M., Ramirez, J., Salas-Gonzalez, D.: Improving MR Brain Image Segmentation Using Self-organising Maps and Entropy-gradient Clustering. Information Sciences 262, pp. 117-136 (2014)

20. Tayefi, M., Esmaeili, H., Karimian, M.S., Zadeh, A.A., Ebrahimi, M., Safarian, M., Nematy, M., Parizadeh, S.M., Ferns, G.A., Ghayour-Mobarhan, M.: The Application of a Decision Tree to Establish the Parameters Associated with Hypertension. Computer Methods and Programs in Biomedicine (2016)

21. Wang, Y., Simon, M.A., Bonde, P., Harris, B.U., Teuteberg, J.J., Kormos, R.L., Antaki, J.F.: Decision Tree for Adjuvant Right Ventricular Support in Patients Receiving a Left Ventricular Assist Device. The Journal of Heart and Lung Transplantation 31(2), 140-149 (2012) 
22. Brims, F., Meniawy, T.M., Duffus, I., de Fonseka, D., Segal, A., Creaney, J., Maskell, N., Lake, R.A., de Klerk, N., Nowak, A.K.: A Novel Clinical Prediction Model for Prognosis in Malignant Pleural Mesothelioma Using Decision Tree Analysis. Journal of Thoracic Oncology: Official Publication of the International Association for the Study of Lung Cancer 11(4), 573-582 (2016)

23. Exarchos, T.P., Tzallas, A.T., Baga, D., Chaloglou, D., Fotiadis, D.I., Tsouli, S., Diakou, M., Konitsiotis, S.: Using Partial Decision Trees to Predict Parkinson's Symptoms: A New Approach for Diagnosis and Therapy in Patients Suffering from Parkinson's Disease. Computers in Biology and Medicine 42(2), 195-204 (2012)

24. Hertz, J., Palmer, R.G., Krogh, O.S.: Introduction to the Theory of Neural Computation. Perseus Publishing (1991)

25. Talbi, E.G.: Metaheuristics: From Design to Implementation. Wiley y Sons, USA (2009)

26. Araujo, A.: Breast cancer diagnosis based on mammary thermography and extreme. Learning Machines (2018)

27. Solarte Martínez, G.R., Soto Mejía, J.A.: Árboles de decisiones en el diagnóstico de enfermedades cardiovasculares. Scientia Et Technica XVI (2011)

28. Gonzalez, F.A.: Modelos de aprendizaje computacional en reumatología. Rev. Colomb. R eumatol. [online] 22(2), 77-78 (2015)

29. Morales, L.: Modelo computacional para la identificación de endofenotipos y clasificación de pacientes con artritis reumatoide a partir de datos genéticos, serológicos y clínicos, utilizando técnicas de inteligencia computacional. Revista Colombiana de Reumatología 22(2), 90-103 (2015)

30. Abad-Grau, M.M.: Aplicación de Redes Bayesianas en el Modelado de un Sistema Experto de Triaje en Servicios de Urgencias Médicas. En: IX Workshop de Investigadores en Ciencias de la Computación, pp. 43-47 (2007)

31. John, J.: Diagnóstico automático del síndrome coronario agudo utilizando un sistema multiagente basado en redes neuronales. Revista Colombiana de Cardiología 24(3), 255-260 (2017)

32. Álvarez, M., Nava, J.M., Rue, M, Quintana, S.: Mortality prediction in head trauma patients: Performance of Glasgow Coma Score and general severity systems. Crit Care Med 26, 142-148 (1998) 